AD/AP-28

Accelerator Development Department

Accelerator Physics Division

BROOKHAVEN NATIONAL LABORATORY

Associated Universities, Inc.

Upton, NY 11973

Accelerator Physics Technical Note No. 28

Emittance Change Due to Linear Coupling Possible Correction Scheme of Emittance Growth

V. Garczynski

September 1991 


\title{
EMITTANCE CHANGE DUE TO LINEAR COUPLING - POSSIBLE CORRECTION SCHEME OF EMITTANCE GROWTH
}

\author{
V. Garczynski \\ Accelerator Development Department \\ Brookhaven National Laboratory
}

September 1991 


\section{Contents}

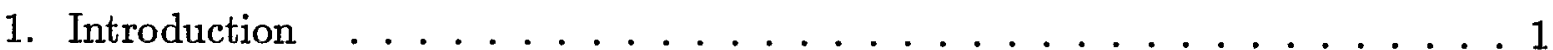

2. Symplecticity and Its Consequences $\ldots \ldots \ldots \ldots$

3. The Beam 4-Ellipsoid $\ldots \ldots \ldots \ldots \ldots$

4. The Case of Single Thin Skew-Quadrupole of Arbitrary Strength . . . . . . 9

5. The Case of $N$ Thin Skew-Quadrupoles Randomly Distributed Around a Ring 11

6. Correction Scheme for Emittance Growth in RHIC (An Example) . . . . . . 13

7. Simple Consequences of the Basic Relations . . . . . . . . . . . . 16

8. Acknowledgments $\ldots \ldots \ldots \ldots \ldots$

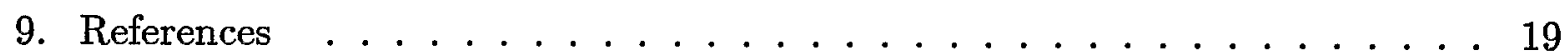




\title{
EMITTANCE CHANGE DUE TO LINEAR COUPLING - POSSIBLE CORRECTION SCHEME OF EMITTANCE GROWTH
}

\author{
V. Garczynski \\ Accelerator Development Department \\ Brookhaven National Laboratory \\ September 1991
}

\section{Introduction}

A coupling between $X$ and $Y$ degrees of freedom due to various sources will be present in RHIC. ${ }^{1,2}$ In this note we shall examine the projected emittances change due to skewquadrupoles randomly distributed around a ring. They will linearly couple the $x$ and $y$ betatron motions, and their effect can be calculated exactly within a model in which skewquadrupole magnets act like point objects of a given strength $q_{k}$ and location $s_{k}, k=1$, $\ldots, N^{3}$. This approximation is justified, (in the machine with distributed parameters), by comparing the length $\ell=0.6 \mathrm{~m}$ of a skew-quadrupole magnet and the length $C=3833.852$ $\mathrm{m}$ of RHIC's circumference, $\ell / C \sim 10^{-4}$.

We shall use the same notations as in our earlier papers on the linear coupling phenomenon $^{4,5}$. Our work can be viewed as an extension of Brown and Servranckx general analysis ${ }^{6}$ of the influence of the linear coupling on the projected emittance. Our aim is to reveal the main driving terms responsible for projected emittance change due to the linear coupling, and to obtain more quantitative results. We also would like to propose a correction scheme for emittance growth in RHIC. In fact we show that the correction scheme for the tune splitting proposed by A.G. Ruggiero, which is perhaps to strong just for this purpose, will correct the projected emittance growth, as well. 


\section{Symplecticity and Its Consequences}

When the transverse $x$ and $y$ betatron motions are linearly coupled the $\left(x, x^{\prime}\right)$ and $\left(y, y^{\prime}\right)$ variables transform jointly when passing from one point, say $s_{0}$, in a ring to another, $s=s_{1}$.

$$
z_{1}=T z_{0}
$$

where

$$
z=\left[\begin{array}{c}
x \\
x^{\prime} \\
y \\
y^{\prime}
\end{array}\right]
$$

and $T$ is $4 \times 4$ real symplectic $4 \times 4$ matrix

$$
T=\left[\begin{array}{ll}
M & n \\
m & N
\end{array}\right]
$$

satisfying the condition

$$
\tilde{T} S T=S,
$$

( $\tilde{T}$ is transpose of $T$ ).

Here $S$ stands for the $4 \times 4$ matrix

$$
S=\left[\begin{array}{ll}
\sigma & 0 \\
0 & \sigma
\end{array}\right]
$$

and

$$
\sigma=\left[\begin{array}{cc}
0 & 1 \\
-1 & 0
\end{array}\right]
$$

is one of the fundamental Pauli matrices. The symplecticity condition (2.4) may be written in two equivalent forms,

$$
1^{\circ} \quad T \bar{T}=1
$$

or

$$
2^{\circ} \quad \bar{T} T=1
$$

where

$$
\bar{T}=-S \tilde{T} S=\left[\begin{array}{cc}
\bar{M} & \bar{m} \\
\bar{n} & \bar{N}
\end{array}\right]=T^{-1},
$$

and the bar denotes a symplectic conjugate of a matrix.

$$
M=\left[\begin{array}{ll}
M_{11} & M_{12} \\
M_{21} & M_{22}
\end{array}\right] \longrightarrow \bar{M}=\left[\begin{array}{cc}
M_{22} & -M_{12} \\
-M_{21} & M_{11}
\end{array}\right]
$$


In terms of the submatrices, the first symplecticity condition, reads

$$
T \bar{T}=\mathbf{1} \longrightarrow \begin{aligned}
& \text { a. } M \bar{M}+n \bar{n}=\mathbf{1}, \\
& \text { b. } N \bar{N}+m \bar{m}=\mathbf{1}, \\
& \text { c. } M \bar{m}+n \bar{N}=\mathbf{0}, \\
& \text { d. } m \bar{M}+N \bar{n}=\mathbf{0} .
\end{aligned}
$$

Using the fact that for any $2 \times 2$ matrix one has

$$
M \bar{M}=|M| \mathbf{1},
$$

one gets from a. and b., respectively, the equalities

$$
|M|+|n|=1
$$

and

$$
|N|+|m|=1 \text {. }
$$

The condition c. yields four independent relations

$$
\begin{aligned}
& \left|\begin{array}{ll}
M_{11} & M_{12} \\
m_{21} & m_{22}
\end{array}\right|+\left|\begin{array}{ll}
n_{11} & n_{12} \\
N_{21} & N_{22}
\end{array}\right|=0, \\
& \left|\begin{array}{ll}
N_{11} & N_{12} \\
n_{11} & n_{12}
\end{array}\right|+\left|\begin{array}{ll}
m_{11} & m_{12} \\
M_{11} & M_{12}
\end{array}\right|=0, \\
& \left|\begin{array}{ll}
M_{21} & M_{22} \\
m_{21} & m_{22}
\end{array}\right|+\left|\begin{array}{ll}
n_{21} & n_{22} \\
N_{21} & N_{22}
\end{array}\right|=0, \\
& \left|\begin{array}{ll}
N_{11} & N_{12} \\
n_{21} & n_{22}
\end{array}\right|+\left|\begin{array}{ll}
m_{11} & m_{12} \\
M_{12} & M_{22}
\end{array}\right|=0 .
\end{aligned}
$$

The last condition, d., is equivalent to, c., upon the symplectic conjugation and, in fact, is irrelevant. Thus altogether one has six independent conditions reducing the number of independent parameters in a $4 \times 4$ symplectic matrix from 16 to $10^{7}$.

The equivalent set of six independent conditions on $T$ follows from the equations

$$
\bar{T} T=\mathbf{1} \longrightarrow \begin{aligned}
& \text { a.' } \bar{M} M+\bar{m} m=\mathbf{1}, \\
& \text { b. } \bar{N} N+\bar{n} n=\mathbf{1}, \\
& \text { d.' } \bar{N} m+\bar{m} N=\mathbf{0},
\end{aligned}
$$


One notices that this set of relations follows from the previous set (2.11) upon the substitions,

$$
\begin{gathered}
M \rightarrow \bar{M}, \\
N \rightarrow \bar{N}, \\
m \rightarrow \bar{n}, \\
n \rightarrow \bar{m} .
\end{gathered}
$$

Hence, applying them to Eqs. (2.13) - (2.15) one gets at once

$$
|M|+|m|=1
$$

and

$$
|N|+|n|=1
$$

and four conditions corresponding to (2.15). We shall not reproduce them here explicitly as they will not be needed in the sequel. Comparing (2.13), (2.14) and (2.18), (2.19) one gets the well known equalities

$$
|m|=|n|
$$

and

$$
|M|=|N|=1-|n|
$$




\section{The Beam 4-Ellipsoid}

When the linear coupling is present one considers instead of two separate ellipses, a single 4-dimensional ellipsoid, (at a point of a ring),

$$
\tilde{z} \sigma^{-1} z=1
$$

where (please, do not confuse with $\sigma$-matrix entering $S$ of (2.6))

$$
\sigma=\left[\begin{array}{cc}
\sigma_{x} & t \\
\tilde{t} & \sigma_{y}
\end{array}\right]
$$

is a symmetric and positive- definite $4 \times 4$ matrix while $\sigma_{x}, \sigma_{y}$ are symmetric, positivedefinite, $2 \times 2$ submatrices representing projections of the beam onto $\left(x, x^{\prime}\right)$ and $\left(y, y^{\prime}\right)$ planes and $t$ represents the coupling. When passing from a point $s=s_{0}$ to another one at $s=s_{1}$ the matrix $\sigma$ transforms as

$$
\sigma_{1}=T \sigma_{0} \tilde{T}
$$

In terms of the subblocks this condition is equivalent to the following ones

$$
\begin{aligned}
& \sigma_{x 1}=M \sigma_{x 0} \tilde{M}+n \sigma_{y 0} \tilde{n}+n \tilde{t_{0}} \tilde{M}+M t_{0} \tilde{n}, \\
& \sigma_{y 1}=N \sigma_{y 0} \tilde{N}+m \sigma_{x 0} \tilde{m}+N \tilde{t} \tilde{m}+m t_{0} \tilde{N},
\end{aligned}
$$

and

$$
t_{1}=n \tilde{t}_{0} \tilde{m}+M \sigma_{x 0} \tilde{m}+n \sigma_{y 0} \tilde{N}
$$

If the initial beam is decoupled i.e., if $t_{0}=0$, these relations simplify

$$
\begin{aligned}
\sigma_{x 1} & =M \sigma_{x 0} \tilde{M}+n \sigma_{y 0} \tilde{n}, \\
\sigma_{y 1} & =N \sigma_{y 0} \tilde{N}+m \sigma_{x 0} \tilde{m}, \\
t_{1} & =M \sigma_{x 0} \tilde{m}+n \sigma_{y 0} \tilde{N} .
\end{aligned}
$$

Denoting the initial projected emittances as $\epsilon_{x 0}, \epsilon_{y 0}$ where

$$
\epsilon_{x 0}^{2}=\left|\sigma_{x 0}\right| \quad, \quad \epsilon_{y 0}^{2}=\left|\sigma_{y 0}\right|
$$

we get for the squares of emittances at the location $s_{1}$

$$
\epsilon_{x 1}^{2}=\left|\sigma_{x 1}\right|=\left|M \sigma_{x 0} \tilde{M}+n \sigma_{y 0} \tilde{n}\right|,
$$

and 


$$
\epsilon_{y 1}^{2}=\left|\sigma_{y 1}\right|=\left|N \sigma_{y 0} \tilde{N}+m \sigma_{x 0} \tilde{m}\right| .
$$

We assume for simplicity that the initial beam ellipses are upright, i.e., corresponding $\sigma_{x 0}, \sigma_{y 0}$ are diagonal

$$
\begin{aligned}
& \sigma_{x 0}=\left[\begin{array}{cc}
\sigma_{11} & 0 \\
0 & \sigma_{22}
\end{array}\right], \\
& \sigma_{y 0}=\left[\begin{array}{cc}
\sigma_{33} & 0 \\
0 & \sigma_{44}
\end{array}\right],
\end{aligned}
$$

and then

$$
\epsilon_{x 0}^{2}=\sigma_{11} \sigma_{22} \quad, \quad \epsilon_{y 0}^{2}=\sigma_{33} \sigma_{44} .
$$

The emittances $\epsilon_{x 1}, \epsilon_{y 1}$ can be found from the formulae (3.11), (3.12) with use of the identity which holds for any real $2 \times 2$ matrices $A$ and $B$

$$
|A+B|=|A|+|B|+\frac{1}{2} \operatorname{Tr}(A \bar{B}+B \bar{A})=|A|+|B|+\left|\begin{array}{ll}
A_{11} & A_{12} \\
B_{21} & B_{22}
\end{array}\right|+\left|\begin{array}{ll}
B_{11} & B_{12} \\
A_{21} & A_{22}
\end{array}\right|
$$

Performing the above calculations we get for the emittances at the location $s_{1}$ the formula

$$
\epsilon_{x 1}^{2}=\epsilon_{x 0}^{2}|M|^{2}+\epsilon_{y 0}^{2}|n|^{2}+\Delta_{x}
$$

and

$$
\epsilon_{y 1}^{2}=\epsilon_{y 0}^{2}|N|^{2}+\epsilon_{x 0}^{2}|m|^{2}+\Delta_{y}
$$

where we denoted

$$
\begin{gathered}
\Delta_{x}=\sigma_{11} \sigma_{33}\left|\begin{array}{ll}
M_{11} & n_{11} \\
M_{21} & n_{21}
\end{array}\right|^{2}+\sigma_{11} \sigma_{44}\left|\begin{array}{ll}
M_{11} & n_{12} \\
M_{21} & n_{22}
\end{array}\right|^{2}+ \\
+\sigma_{22} \sigma_{33}\left|\begin{array}{ll}
M_{12} & n_{11} \\
M_{22} & n_{21}
\end{array}\right|^{2}+\sigma_{22} \sigma_{44}\left|\begin{array}{ll}
M_{12} & n_{12} \\
M_{22} & n_{22}
\end{array}\right|^{2}
\end{gathered}
$$

and

$$
\begin{aligned}
\Delta_{y} & =\sigma_{11} \sigma_{33}\left|\begin{array}{ll}
m_{11} & N_{11} \\
m_{21} & N_{21}
\end{array}\right|^{2}+\sigma_{11} \sigma_{44}\left|\begin{array}{ll}
m_{11} & N_{12} \\
m_{21} & N_{22}
\end{array}\right|^{2}+ \\
& +\sigma_{22} \sigma_{33}\left|\begin{array}{ll}
m_{12} & N_{11} \\
m_{22} & N_{21}
\end{array}\right|^{2}+\sigma_{22} \sigma_{44}\left|\begin{array}{ll}
m_{12} & N_{12} \\
m_{22} & N_{22}
\end{array}\right|^{2} .
\end{aligned}
$$

From the symplecticity conditions (2.15) follows the equality

$$
\Delta_{x}=\Delta_{y}=\Delta \text {. }
$$


Assuming that the beam and machine ellipses match perfectly, i.e., that beam and machine ellipses coincide, we get the equalities

$$
\begin{aligned}
& \sigma_{11}=\epsilon_{x 0} \beta_{x}, \\
& \sigma_{22}=\epsilon_{x 0} \beta_{x}^{-1}, \\
& \sigma_{33}=\epsilon_{y 0} \beta_{y}, \\
& \sigma_{44}=\epsilon_{y 0} \beta_{y}^{-1} .
\end{aligned}
$$

Taking into account the formula (2.20), (2.21), we get our basic relations for projected emittances

$$
\epsilon_{x 1}^{2}=(1-|n|)^{2} \epsilon_{x 0}^{2}+|n|^{2} \epsilon_{y 0}^{2}+\Delta
$$

and

$$
\epsilon_{y 1}^{2}=(1-|n|)^{2} \epsilon_{y 0}^{2}+|n|^{2} \epsilon_{x 0}^{2}+\Delta
$$

and

$$
\begin{aligned}
\Delta=\epsilon_{x 0} \epsilon_{y 0} & \left(\beta_{x} \beta_{y}\left|\begin{array}{ll}
M_{11} & n_{11} \\
M_{21} & n_{21}
\end{array}\right|^{2}+\beta_{x} \beta_{y}^{-1}\left|\begin{array}{ll}
M_{11} & n_{12} \\
M_{21} & n_{22}
\end{array}\right|^{2}+\right. \\
& \left.+\beta_{x}^{-1} \beta_{y}\left|\begin{array}{ll}
M_{12} & n_{11} \\
M_{22} & n_{21}
\end{array}\right|^{2}+\beta_{x}^{-1} \beta_{y}^{-1}\left|\begin{array}{ll}
M_{12} & n_{12} \\
M_{22} & n_{22}
\end{array}\right|^{2}\right) .
\end{aligned}
$$

One notices that, by subtracting projected emittances squares, the following general formula holds

$$
\epsilon_{x 1}^{2}-\epsilon_{y 1}^{2}=(1-2|n|)\left(\epsilon_{x 0}^{2}-\epsilon_{y 0}^{2}\right) .
$$

Moreover, positive definiteness of the matrix $\sigma$ and its submatrices $\sigma_{x}, \sigma_{y}$ implies the general inequalities

$$
\epsilon_{x 1} \geq|1-\operatorname{det} n| \epsilon_{x 0}+|\operatorname{det} n| \epsilon_{y 0},
$$

and

$$
\epsilon_{y 0} \geq|1-\operatorname{det} n| \epsilon_{y 0}+|\operatorname{det} n| \epsilon_{x 0} .
$$

These formulas were obtained and their consequences were studied by Brown and Servranckx. We go beyond their analysis by considering in detail both, $|n|$ and $\Delta$, within our discrete model. We calculate these terms in two cases of the linear coupling produced by: A. Single skew-quadrupole of an arbitrary strength $q$ at a location $s_{1}$ in a ring, 
B. An arbitrary number $N$ of thin skew-quadrupoles of small strengths $q_{k}$ located at $s_{k}$, $k=1, \ldots, N$.

In the first case our results are exact, in the second they are valid up to the second order in the $q$ 's.

We find the main driving terms responsible for emittance change, and study an influence on it of the tune splitting correction scheme. We also propose a scheme for correction of the emittance growth in case of RHIC. 


\section{The Case of Single Thin Skew-Quadrupole of Arbitrary Strength}

Let a thin skew-quadrupole of length $\ell$ and of strength $q$ be located at $s=s_{1}$ in an otherwise perfect lattice. The transfer matrix $T$ connecting the point of observation at $s=s_{0}=0$ and the point $s=s_{1}$ is given by the product of the relevant transfer matrices

$$
\begin{aligned}
& T\left(s_{1,0}\right)=T_{S Q}\left(s_{1}\right) T^{(0)}\left(s_{1,0}\right)= \\
& =\mathcal{B}^{-1}\left(s_{1}\right) \stackrel{\circ}{T}_{S Q}\left(s_{1}\right) \stackrel{\circ}{T}^{(0)}\left(s_{1,0}\right) \mathcal{B}(0),
\end{aligned}
$$

where $\stackrel{\circ}{T}_{S Q}\left(s_{1}\right)$ is the transfer matrix of the thin skew-quadrupole (in the circular representation denoted by o above $T_{S Q}$ )

$$
\stackrel{\circ}{T}_{S Q}\left(s_{1}\right)=\left[\begin{array}{llll}
1 & 0 & 0 & 0 \\
0 & 1 & q & 0 \\
0 & 0 & 1 & 0 \\
q & 0 & 0 & 1
\end{array}\right],
$$

and

$$
q=\left.\left(\beta_{x} \beta_{y}\right)^{1 / 2} \frac{\ell}{\rho} a_{1}\right|_{s_{1}}
$$

while the $\stackrel{\circ}{T}^{(0)}\left(s_{1,0}\right)$ represents transfer matrix of a perfect lattice

$$
\stackrel{\circ}{T}^{(0)}\left(s_{1,0}\right)=\left[\begin{array}{cc}
R\left(\psi_{x}\right) & 0 \\
0 & R\left(\psi_{y}\right)
\end{array}\right],
$$

where $R(\psi)$ is a usual rotation matrix

$$
R(\psi)=\left[\begin{array}{rr}
\cos \psi & \sin \psi \\
-\sin \psi & \cos \psi
\end{array}\right]
$$

and $\psi_{x}, \psi_{y}$ are phase advances given by the standard expressions

$$
\psi_{x}=\int_{0}^{s_{1}} \frac{d s}{\beta_{x}}, \quad \psi_{y}=\int_{0}^{s_{1}} \frac{d s}{\beta_{y}},
$$

with $\beta_{x}, \beta_{y}$ being $\beta$-functions of the perfect machine. They, together with $\alpha_{x}, \alpha_{y}$ lattice functions enter the matrix $\mathcal{B}$

where

$$
\mathcal{B}=\left[\begin{array}{cc}
\mathcal{B}_{x} & 0 \\
0 & \mathcal{B}_{y}
\end{array}\right]
$$

$$
\mathcal{B}_{x}=\left[\begin{array}{cc}
\beta_{x}^{1 / 2} & 0 \\
\alpha_{x} \beta_{x}^{-1 / 2} & \beta_{x}^{1 / 2}
\end{array}\right],
$$


and similar for $\mathcal{B}_{y}$.

Performing the operations prescribed by the formula (4.1) we get the results

$$
T\left(s_{1,0}\right)=\left[\begin{array}{ll}
M\left(s_{1,0}\right) & n\left(s_{1,0}\right) \\
m\left(s_{1,0}\right) & N\left(s_{1,0}\right)
\end{array}\right] .
$$

where

$$
M\left(s_{1,0}\right)=\left[\begin{array}{cc}
\left(\beta_{x}\left(s_{1}\right) / \beta_{x}\right)^{1 / 2} \cos \psi_{x} & \left(\beta_{x}\left(s_{1}\right) \beta_{x}\right)^{1 / 2} \sin \psi_{x} \\
\cdots & \cdots
\end{array}\right]
$$

and

$$
n\left(s_{1,0}\right)=q\left[\begin{array}{cc}
0 & 0 \\
\left(\beta_{x}\left(s_{1}\right) \beta_{y}\right)^{1 / 2} \cos \psi_{y} & \left(\beta_{y} / \beta_{x}\left(s_{1}\right)\right)^{1 / 2} \sin \psi_{y}
\end{array}\right]
$$

and

$$
N\left(s_{1,0}\right)=M\left(s_{1,0}\right)_{\mid x \leftrightarrow y},
$$

and

$$
m\left(s_{1,0}\right)=n\left(s_{1,0}\right)_{\mid x \rightarrow y} .
$$

We omit here the terms in lower row of the matrix $M\left(s_{1,0}\right)$ as they are irrelevant when multiplied by zeros appearing in the upper row of the matrix $n\left(s_{1,0}\right)$, in calculating the $\Delta$. According to the formula (3.25) we get the result

$$
\Delta=q^{2} \epsilon_{x 0} \epsilon_{y 0}
$$

Moreover, taking into account that the determinant $|n|$ vanishes in this case one obtains for the projected emittances, at the location $s=s_{1}$, the formulae

$$
\begin{aligned}
& \epsilon_{x 1}^{2}=\epsilon_{x 0}^{2}+q^{2} \epsilon_{x 0} \epsilon_{y 0} \\
& \epsilon_{y 1}^{2}=\epsilon_{y 0}^{2}+q^{2} \epsilon_{x 0} \epsilon_{y 0} .
\end{aligned}
$$

We see that emittances grow equally fast, in both $\left(x, x^{\prime}\right)$ and $\left(y, y^{\prime}\right)$ planes, when a beam passes through a skew-quadrupole magnet. One may notice in passing that a flat beam $\left(\epsilon_{y 0}=0\right)$ remains such immediately after crossing a single skew-quadrupole, $\left(\epsilon_{x 1}=\epsilon_{x 0}, \epsilon_{y 1}=0\right)$. 


\section{The Case of $N$ Thin Skew-Quadrupoles Randomly Distributed Around a Ring}

In this case we consider a single turn transfer matrix $T(C, 0)$ at the point of observation $s=s_{0}=0$, calculated up to the second order in the $q$ 's. We have in this case for the determinant of $n$

$$
|n|=\sum_{1 \leq r<s \leq N} q_{r} q_{s} \sin \left(\mu_{x}^{r}-\mu_{x}^{r}\right) \sin \left(\mu_{y}^{s}-\mu_{y}^{r}\right)+O\left(q^{4}\right)
$$

and for the $\Delta$ we get

$$
\Delta=\epsilon_{x 0} \epsilon_{y 0} \sum_{r, s=1}^{N} q_{r} q_{s} \cos \left(\mu_{x}^{s}-\mu_{x}^{r}\right) \cos \left(\mu_{y}^{s}-\mu_{y}^{r}\right)+O\left(q^{4}\right)
$$

Taking into account that, in the average, we have

$$
\begin{aligned}
<|n|> & =0 \\
<|n|^{2}> & =\frac{1}{8}<q^{2}>N(N-1) \simeq \frac{1}{8} G_{0}^{4} \\
<\Delta> & =\frac{1}{2} G_{0}^{2} \epsilon_{x 0} \epsilon_{y 0}
\end{aligned}
$$

where we denoted

$$
G_{0}^{2}=N<q^{2}>
$$

we obtain for the average projected emittance squares

$$
<\epsilon_{x 1}^{2}>=\epsilon_{x 0}^{2}+\frac{1}{8} G_{0}^{4}\left(\epsilon_{x 0}^{2}+\epsilon_{y 0}^{2}\right)+\frac{1}{2} G_{0}^{2} \epsilon_{x 0} \epsilon_{y 0} \geq \epsilon_{x 0}^{2}
$$

and

$$
<\epsilon_{y 1}^{2}>=\epsilon_{y 0}^{2}+\frac{1}{8} G_{0}^{4}\left(\epsilon_{x 0}^{2}+\epsilon_{y 0}^{2}\right)+\frac{1}{2} G_{0}^{2} \epsilon_{x 0} \epsilon_{y 0} \geq \epsilon_{y 0}^{2}
$$

Assuming that

$$
G_{0}= \begin{cases}0.25, & \text { for RHIC } \\ 0.5-1.0, & \text { for SSC }\end{cases}
$$

we get the estimates for average projected emittance squares in case of RHIC

$$
\begin{aligned}
& <\epsilon_{x 1}^{2}>=\epsilon_{x 0}^{2}+5 \times 10^{-4}\left(\epsilon_{x 0}^{2}+\epsilon_{y 0}^{2}\right)+3 \times 10^{-2} \epsilon_{x 0} \epsilon_{y 0} \\
& <\epsilon_{y 1}^{2}>=\epsilon_{y 0}^{2}+5 \times 10^{-4}\left(\epsilon_{x 0}^{2}+\epsilon_{y 0}^{2}\right)+3 \times 10^{-2} \epsilon_{x 0} \epsilon_{y 0}
\end{aligned}
$$


and even larger effect for the case of SSC,

$$
\begin{aligned}
& <\epsilon_{x 1}^{2}>=\epsilon_{x 0}^{2}+78 \times 10^{-4}\left(\epsilon_{x 0}^{2}+\epsilon_{y 0}^{2}\right)+12 \times 10^{-2} \epsilon_{x 0} \epsilon_{y 0} \\
& <\epsilon_{y 1}^{2}>=\epsilon_{y 0}^{2}+78 \times 10^{-4}\left(\epsilon_{x 0}^{2}+\epsilon_{Y 0}^{2}\right)+12 \times 10^{-2} \epsilon_{x 0} \epsilon_{y 0} .
\end{aligned}
$$




\section{Correction Scheme for Emittance Growth in RHIC (An Exam- ple)}

It is interesting to examine first how the tune splitting correction influences the emittance growth produced by linear coupling. We use an intersection point as the observation point $s=0$ where the correction is carried out. In order to see this we must disclose the main driving terms responsible for emittance growth. They can be most easily seen by first writing the determinant $|n|$ and the term $\Delta$ as

$$
|n|=d_{s s}^{(1)} d_{c c}^{(1)}-d_{s c}^{(1)} d_{c s}^{(1)}+O\left(q^{4}\right)=d_{s c}^{(2)}-d_{c s}^{(2)}+O\left(q^{4}\right),
$$

and

$$
\Delta=\epsilon_{x 0} \epsilon_{y 0}\left[\left(d_{c c}^{(1)}\right)^{2}+\left(d_{c s}^{(1)}\right)^{2}+\left(d_{s c}^{(1)}\right)^{2}+\left(d_{s s}^{(1)}\right)^{2}\right]+O\left(q^{4}\right)
$$

where $d^{(1)}, d^{(2)}$ are the first and the second order driving terms, [cf. 4, formulae (2.7) and (2.8)].

On correction of the tune splitting, up to the second order in the $q$ 's, one requires that the following condition, among others, holds

$$
d_{s c}^{(2)}-d_{c s}^{(2)}=0
$$

One sees that this amounts to vanishing of the determinant $|n|$, and has effect on the emittance growth as it is seen from the formulae (3.23) and (3.24).

One has then after the tune splitting correction

$$
\epsilon_{x 1_{\left.\right|_{\Delta \nu=0}}^{2}}^{2}=\epsilon_{x 0}^{2}+\Delta_{\left.\right|_{\Delta \nu=0}}
$$

and

$$
\epsilon_{y 1_{\mid \Delta \nu=0}}=\epsilon_{y 0}^{2}+\Delta_{\left.\right|_{\Delta \nu=0}}
$$

It is clear from the formula (6.2) that the projected emittance would stay unchanged, up to the fourth order in the $q$ 's, if one corrects, additionally, all the first order driving terms

$$
\left[\begin{array}{l}
d_{s s}^{(1)} \\
d_{s c}^{(1)} \\
d_{c s}^{(1)} \\
d_{c c}^{(1)}
\end{array}\right]=\sum_{r=1}^{N} q_{r}\left[\begin{array}{c}
\sin \mu_{x}^{r} \sin \mu_{y}^{r} \\
\sin \mu_{x}^{r} \cos \mu_{y}^{r} \\
\cos \mu_{x}^{r} \sin \mu_{y}^{r} \\
\cos \mu_{x}^{r} \cos \mu_{y}^{r}
\end{array}\right]=0
$$


It is clear from the formulae (6.1) and (6.2) that both, $|n|$ and $\Delta$, vanish in this case, up to the fourth order in the $q$ 's. In fact, the whole matrix $n$ vanish in this case, and the projected emittance stay unchanged, at the point of correction, up to the fourth order in the $q$ 's

$$
\epsilon_{x 1_{l_{n}=0}}=\epsilon_{x 0}+O\left(q^{4}\right)
$$

and

$$
\epsilon_{y 1_{\mid n=0}}=\epsilon_{y 0}+O\left(q^{4}\right)
$$

As in the case of the tune splitting correction scheme for RHIC, the correction of the combinations of the second order driving terms can be done most effectively in the insertions. One can achieve both the tune splitting correction and the emittance growth correction by requiring, at the crossing points in RHIC, the conditions

$$
\begin{aligned}
& d_{s s}^{(1)}=0, \\
& d_{c s}^{(1)}=0, \\
& d_{s c}^{(1)}=0, \\
& d_{c c}^{(1)}=0, \\
& d_{c c}^{(2)}+d_{s s}^{(2)}=0, \\
& \check{d}_{c c}^{(2)}+\check{d}_{s s}^{(2)}=0 .
\end{aligned}
$$

In order to make the paper rather self contained we quote the relevant combinations of the second order driving terms appearing in the above formulae

$$
d_{c c}^{(2)}+d_{s s}^{(2)}=\sum_{1 \leq r<s \leq N} q_{r} q_{s} \sin \left(\mu_{y}^{s}-\mu_{y}^{r}\right) \cos \left(\mu_{x}^{s}-\mu_{x}^{r}\right)
$$

and

$$
\breve{d}_{c c}^{(2)}+\breve{d}_{s s}^{(2)}=\sum_{1 \leq r<s \leq N} q_{r} q_{s} \sin \left(\mu_{x}^{s}-\mu_{x}^{r}\right) \cos \left(\mu_{y}^{s}-\mu_{y}^{r}\right) .
$$

One sees that the proposed correction scheme for emittance growth will remove the tune splitting at the same time. The price for this comfort is rather small - six instead of five conditions needed to remove just the tune splitting. Because, in RHIC, correctors 
in the arcs are $\sim 90^{\circ}$ apart in each plane, it is not possible to correct locally the above combinations of the second order driving terms. One must place the relevant correctors in the insertions.

We conclude with the statement that the correction scheme proposed by A.G. Ruggiero ${ }^{3}$ for the tune splitting will correct, same time, the projected emittance growth at the crossing points. 


\section{Simple Consequences of the Basic Relations}

Following Brown and Servranckx we shall examine few simple consequences of the basic relations (3.23), (3.24) and (3.25) augmented by our knowledge of the determinant $|n|$, and the term $\Delta$ appearing there.

1. If $\epsilon_{x 0}=\epsilon_{y 0}$, then $\epsilon_{x 1}=\epsilon_{y 1}$ everywhere in a ring independently of the coupling, and

$$
\epsilon_{x 1}^{2}=\epsilon_{y 1}^{2}=\left[(1-|n|)^{2}+|n|^{2}\right] \epsilon_{x 0}^{2}+\Delta .
$$

2. If $|n|=\frac{1}{2}$ at some point $s=s_{1}$ in a ring, then, at this point

$$
|M|=|N|=|m|=\frac{1}{2}
$$

and

$$
\epsilon_{x 1}^{2}=\epsilon_{y 1}^{2}=\frac{1}{4}\left(\epsilon_{x 0}^{2}+\epsilon_{y 0}^{2}\right)+\Delta .
$$

3. If $|n|=0$ at some point $s=s_{1}$, then at this point

$$
\epsilon_{x 1}^{2}=\epsilon_{x 0}^{2}+\Delta_{|| n \mid=0}
$$

and

$$
\epsilon_{y 1}^{2}=\epsilon_{y 0}^{2}+\Delta_{|| n \mid=0}
$$

and, obviously

$$
\epsilon_{x 1}^{2}-\epsilon_{y 1}^{2}=\epsilon_{x 0}^{2}-\epsilon_{y 0}^{2}
$$

4. If $|n|=1$ at some point $s=s_{1}$, then

$$
\epsilon_{x 1}^{2}=\epsilon_{y 0}^{2}+\Delta_{|| n \mid=1}
$$

and

$$
\epsilon_{y 1}^{2}=\epsilon_{x 0}^{2}+\Delta_{|| n \mid=1}
$$

and, in this case, the opposite is true

$$
\epsilon_{x 1}^{2}-\epsilon_{y 1}^{2}=-\left(\epsilon_{x 0}^{2}-\epsilon_{y 0}^{2}\right) .
$$


5. If $\epsilon_{x 0} \neq 0, \epsilon_{y 0}=0$ (flat beam in the horizontal plane at $s_{0}=0$ ) then $\sigma_{33}=$ $\sigma_{44}=0$, and $\Delta=0$, and

$$
\begin{aligned}
& \epsilon_{x 1}^{2}=(1-|n|)^{2} \epsilon_{x 0}^{2} \\
& \epsilon_{y 1}^{2}=|n|^{2} \epsilon_{x 0}^{2} .
\end{aligned}
$$

5a. If in addition to the previous situation, at some point $s=s_{1}$ the inequality holds

$$
0 \leq|n| \leq 1
$$

then

$$
\begin{aligned}
& \epsilon_{x 1}=(1-|n|) \epsilon_{x 0}<\epsilon_{x 0} \\
& \epsilon_{y 1}=|n| \epsilon_{x 0}<\epsilon_{x 0}
\end{aligned}
$$

and, as the result

$$
\epsilon_{x 1}+\epsilon_{y 1}=\epsilon_{x 0}
$$

5b. If, however, at $s=s_{1}$, the inequality holds

$$
|n|<0
$$

then

$$
\begin{aligned}
& \epsilon_{x 1}=(1-|n|) \epsilon_{x 0}>\epsilon_{x 0} \\
& \epsilon_{y 1}=-|n| \epsilon_{x 0}
\end{aligned}
$$

and, at this point

$$
\epsilon_{x 1}-\epsilon_{y 1}=\epsilon_{x 0}
$$

5c. Finally, if at the point $s=s_{1}$, the inequality holds

$$
|n|>1 \text {, }
$$

then

$$
\begin{aligned}
& \epsilon_{x 1}=-(1-|n|) \epsilon_{x 0}, \\
& \epsilon_{y 1}=|n| \epsilon_{x 0}>\epsilon_{x 0},
\end{aligned}
$$

and we have, at this point, an opposite situation

$$
\epsilon_{x 1}-\epsilon_{y 1}=-\epsilon_{x 0} .
$$




\section{Acknowledgments}

I would like to thank Sandro Ruggiero for suggesting the problem of emittance change due to the linear coupling. I thank Fritz Dell and Mark Rhoades-Brown for polishing, or rather "englishing" of my Polish-English. 


\section{References}

1. E.D. Courant and H.S. Snyder, Ann Phys 3, 1 (1958).

2. D.A. Edwards and L.C. Teng, IEEE Trans. Nucl. Sci., Vol. NS-20, No. 3, p. 885 (1973), see also

L.C. Teng, "Concerning $N$-Dimensional Coupled Motions", Fermilab Report FN-229 (1971), and "Coupled Transverse Motion", FNAL-TM-1556 (1989).

3. A.G. Ruggiero, "The Problem of Linear Coupling - Parts I, II, III," Talks at Accelerator Physics Division Meetings, May 16, 23 and 30 (1991), BNL.

4. V. Garczynski, "Beta-Function Distortions Due to Linear Coupling", BNL AD/AP Technical Note No. 24, (August 1991).

5. V. Garczynski, "The Tune Shift Due to Linear Coupling", BNL AD/AP Technical Note No. 25, (August 1991).

6. K.L. Brown and Roger V. Servranckx, "Cross-Plane Coupling and its Effect on Projected Emittance", SLAC-PUB-4679 (1989), and references given there.

7. H. Weyl, "The Classical Groups", 2nd Ed., Chapter VI. Princeton University Press, Princeton, 1946. 